\title{
Pulsational Amplitudes of Cepheids - Their Application to Reveal Companions
}

\author{
László Szabados \\ Konkoly Observatory, P.O. Box 67, H-1525 Budapest XII, Hungary
}

\begin{abstract}
Two parameters are defined involving the observed (peakto-peak) amplitudes in $U, B, V$, and $R$ photometric bands as well as that of the radial velocity variation. They are used to detect companions to Cepheids for a sample about 300 Galactic Cepheids.
\end{abstract}

The high incidence of binaries among Cepheids (Szabados 1995) is remarkable, since the companions can distort various relationships valid for Cepheids, and/or increase the scatter of these relations. Some results of a major survey based on the Johnson and Kron-Cousins $U, B, V$, and $R$ photometric amplitudes and the peak-to-peak amplitude of the radial velocity variations for a sample involving about 300 Cepheids in our Galaxy are presented here. In order to reveal companions to Cepheids, two parameters have been introduced:

1. The amplitude ratio $\left(A R=A_{\mathrm{RV}} / A_{\mathrm{B}}\right)$ which is the radial velocity amplitude divided by the photometric amplitude in the $B$-band. This parameter has been defined by Szabados (1993).

2. The "slope" parameter which differs from the previously defined one (Szabados 1993). Here the slope is the steepness of the line $A_{\lambda} / A_{B}$ vs. $1 / \lambda$ (the wavelength is expressed in micrometers). The line is determined by a least squares fit applied to the four data points corresponding to the $U, B, V$, and $R$ bands. The idea of plotting the normalized amplitudes as a function of $1 / \lambda$ was conceived by Fernie (1979). The slope is sensitive to both blue and red companions.

The $A R$ will be lowered by a companion because the amplitude of the brightness variation is decreased when an additional constant light is present. However, Balona \& Stobie $(1979 a, b)$ pointed out that this amplitude ratio is sensitive to the pulsation mode: for Cepheids pulsating in the first overtone the $A R$ is about 1.4 times larger than for the fundamental mode pulsators. This feature has been confirmed by the observations, so the $A R$ value alone is not indicative of binarity. Using the available observations of Galactic beat Cepheids, the following $A R$ values have been obtained for the individual pulsation modes: fundamental mode: $A R_{0}=29.2 \pm 3.3(\mathrm{n}=11)$; first overtone: $A R_{1}=40.0 \pm 4.9$ $(\mathrm{n}=10)$. Their ratio, 1.37 , is in a good agreement with the theoretical value.

The slope vs. $\log P$ diagram (Fig. 1) shows the following noteworthy features:

- Cepheids with hot companions have anomalously low values of the slope.

- The beat Cepheids indicate that the slope is a characteristic property of the star which is independent of the mode of pulsation, because the value of the slope is practically identical for both excited modes.

- The linear increase of the slope towards longer pulsation periods is remarkable. 


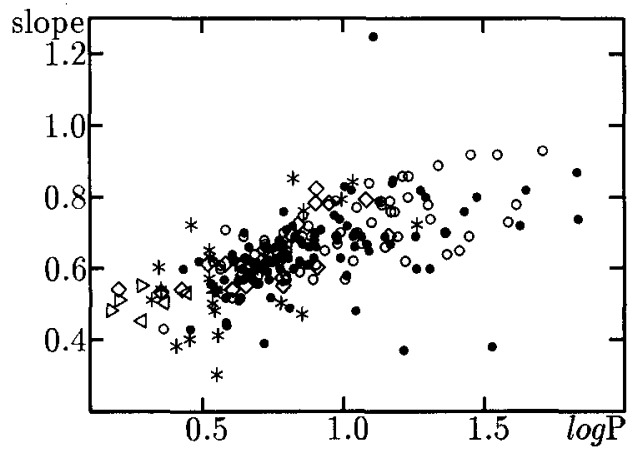

Figure 1. The slope parameter vs. $\log P$. Legend: $\circ:$ normal amplitude Cepheid without known companion; • : normal amplitude Cepheid belonging to binary (or multiple) system; $\diamond:$ s-Cepheid without known companion; * : s-Cepheid with known companion(s); $\triangleright$ : beat Cepheid, first overtone; $\triangleleft:$ beat Cepheid, fundamental mode pulsation. All smallest values of the slope represent binary Cepheids.

Since both methods indicate a companion, one suspects that four Cepheids previously not studied from the point of view of duplicity belong to binary systems: UZ Cas, VW Cas, and V495 Cyg have hot companions, while V520 Cyg has a secondary redder than the Cepheid component.

The study of the Galactic double-mode Cepheids revealed that the longer periods correspond to dominance of first overtone pulsation. In the case of $\mathrm{AX}$ Velorum and $\mathrm{BD}-10^{\circ} 4669$, the two beat Cepheids in the Galaxy whose first overtone oscillation is more developed than the fundamental mode, the $R_{21}$ Fourier-parameter is larger for the oscillation mode with larger amplitude, contrary to the other double-mode Cepheids. Validity of these findings should be confirmed by studying the much larger sample of beat Cepheids discovered in the Magellanic Clouds during the MACHO, EROS, and OGLE projects.

Acknowledgments. Financial support through the AKP 97-58 2,2 and OTKA T022946, T029013 research grants is gratefully acknowledged.

\section{References}

Balona L. A. \& Stobie R. S. 1979a, MNRAS, 189, 627

Balona L. A. \& Stobie R. S. 1979b, MNRAS, 189, 649

Fernie J. D. 1979, PASP, 91, 67

Szabados L. 1993, in Proc. IAU Coll. 139, New Perspectives on Stellar Pulsation and Pulsating Variable Stars, ed. J. M. Nemec \& J. M. Matthews (Cambridge: Cambridge University Press), 406

Szabados L. 1995, in ASP Conf. Ser. Vol. 83, Astrophysical Application of Stellar Pulsation, Proc. IAU Coll. No. 155, ed. R. S. Stobie \& P. A. Whitelock (San Francisco: ASP), 357 